\title{
Tracheostomy among paediatric patients: Our experiences at a tertiary care teaching hospital in Eastern India
}

\author{
Santosh Kumar Swain, Mahesh Chandra Sahu², Jasashree Choudhury³, Biplob Bhattacharyya' \\ 'Department of Otorhinolaryngology, IMS and SUM Hospital, Siksha "O" Anusandhan University, K8, Kalinganagar, \\ Bhubaneswar, Odisha, India \\ "Medical Research Laboratory, IMS and SUM Hospital, Siksha "O" Anusandhan University, K8, Kalinganagar, \\ Bhubaneswar, Odisha, India \\ ${ }^{3 P}$ ediatric Intensive Care Unit, Department of Paediatrics, IMS and SUM Hospital, Siksha "O" Anusandhan University, K8, \\ Kalinganagar, Bhubaneswar, Odisha, India
}

\section{ABSTRACT}

Introduction: Tracheostomy is a surgical procedure that has been performed since ancient times. It is a commonly used life-saving surgical procedure among critically ill patients. Children with airway compromise often need tracheostomy.

Aim of the study: There are numerous research papers published for adult tracheostomy with its indications, operative technique, and complications, but the literature for paediatric tracheostomy is much scarcer. Here, we attempt to analyse the indications, complications, and outcomes of tracheostomy among paediatric patients at a tertiary care teaching hospital in Eastern India.

Material and methods: A retrospective study was done among the children who had undergone tracheostomy between May 2008 and June 2018. The data on age, gender, indications, and complications over a 10-year period were analysed. In this study, the children with tracheostomy were classified into: prolonged intubation, upper airway obstruction, neurological diseases, craniofacial anomalies, trauma, and vocal cord paralysis. Children were included in the prolonged intubation category if they needed ventilator support.

Results: There were 88 tracheostomies done among the children in the study period. There were 47 male children (53.40\%) and 41 females (46.59\%). Age for tracheostomy ranged from seven months to sixteen years, and the mean age of tracheostomy was 8.8 years. The most common indication for tracheostomy was prolonged ventilation $(72.27 \%)$ followed by airway obstruction (22.72\%). Pulmonary pathology was the main cause for prolonged ventilation.

Conclusions: Advanced technique of paediatric anaesthesia and increased awareness for vaccination among children increased the changing indication for tracheostomy from emergency to more elective procedure. Standardisation of the procedure, timing, and exact indications are helping to reduce the mortality related to tracheostomy among paediatric patients.

\section{KEY WORDS:}

tracheostomy, paediatric patients, prolonged ventilation, laryngotracheal stenosis.

\section{ADDRESS FOR CORRESPONDENCE:}

Santosh Kumar Swain, Department of Otorhinolaryngology, IMS and SUM Hospital, Siksha "O" Anusandhan University, K8, Kalinganagar, 751003 Bhubaneswar, Odisha, India, e-mail: santoshvoltaire@yahoo.co.in 


\section{INTRODUCTION}

Tracheostomy is a surgical procedure that has been performed since ancient times. The first tracheostomy is credited to Asclepiades (100 BC), who made an opening of the trachea in a stridor patient, but the outcome of that tracheostomy is unknown [1]. Paul d'Egine documented the first successful tracheostomy in the $16^{\text {th }}$ century. In the $19^{\text {th }}$ century, Armand Trousseau popularised the tracheostomy procedure as a life-saving surgery during a diphtheria epidemic. In the $20^{\text {th }}$ century, Chevalier Jackson described the technique of tracheostomy that we are performing today [1]. Tracheostomy is a commonly performed life-saving surgical procedure among critically ill patients. Children with airway compromise often need tracheostomy. Tracheostomy is a more challenging surgical procedure among children than adults because it is associated with high morbidity and mortality. The risk of complications for tracheostomy is greater among the paediatric group [2].

The indications for tracheostomy among paediatric patients have changed significantly in the last few decades. Initially, upper airway obstructions due to infectious causes comprised the majority of aetiologies for tracheostomy among children [3]. Nowadays the main reasons for tracheostomy in paediatric patients are prolonged ventilation, upper airway obstruction caused by craniofacial malformations, laryngotracheal stenosis, trauma, and neurological disorders associated with hypoventilations [4]. There are numerous research papers published for adult tracheostomy with its indications, operative technique, and complications, but the literature for paediatric tracheostomy is much scarcer. There is always a need for standard guidelines for standard protocols for paediatric tracheostomy.

The aim of this present study is to analyse the indications and complications of tracheostomy among the paediatric group at a tertiary care teaching hospital in Eastern India in the present scenario.

\section{MATERIAL AND METHODS}

This is a retrospective study of tracheostomies performed among paediatric patients at a tertiary care teaching hospital in Eastern India between May 2008 and June 2018. There were 88 children identified who had undergone tracheostomy. Inpatient and outpatient charts of all the patients in this study were analysed. This study was approved by the Institutional Ethical Committee (IEC). The age of the children of this study was divided into neonates ( $<28$ days), infants (up to 1 to 2 year), pre-school (2-6 years), school going (7-9 years), and older children/adolescents (10-16 years). In this study, the children with tracheostomy were classified into: prolonged intubation, upper airway obstruction, neurological diseases, craniofacial anomalies, trauma, and vocal cord paralysis. Children were included in the prolonged intubation category if they needed ventilator support. The upper airway obstruction category had a history of functional or anatomical airway obstruction (e.g. subglottic stenosis, tracheobronchial malacia, severe degree of laryngomalacia, and severe sleep apnoea syndrome). Children with bilateral vocal cord paralysis regardless of cause were included in the bilateral vocal cord paralysis group (Fig. 1). Children with cerebral palsy and hypotonia, obstructive sleep apnoea syndrome, or recurrent pneumonia were included in the neurological category. If the children needed ventilator support following trauma or spinal cord injury, they were included in the trauma group.

All the tracheostomies were carried out by senior ear, nose, and throat (ENT) surgeons in our hospital with the presence of an anaesthetist and a paediatrician in a paediatric intensive care unit (PICU) or paediatric emergency unit. All the tracheostomies among paediatric patients were performed under general anaesthesia. Informed consent, including death on the table in severe conditions, were obtained. A standard procedure for tracheostomy was used in all cases. A horizontal skin incision was made, and once the trachea was visible, a vertical incision was made on the trachea. Two parallel stay sutures were kept on both sides of the incision of the trachea and an appropriate size of tracheostomy tube was inserted. Stomal maturation sutures were applied from the trachea to the skin. Tracheostomy tubes were changed bedside after three days of operation. Detailed parameters such as age, demographics, indications for tracheostomy, co-morbidities, hospital stay, decannulation, trachea-cutaneous fistulas, and other complications were documented. Any death that occurred on the operation table during surgery was considered as mortality due to tracheostomy.

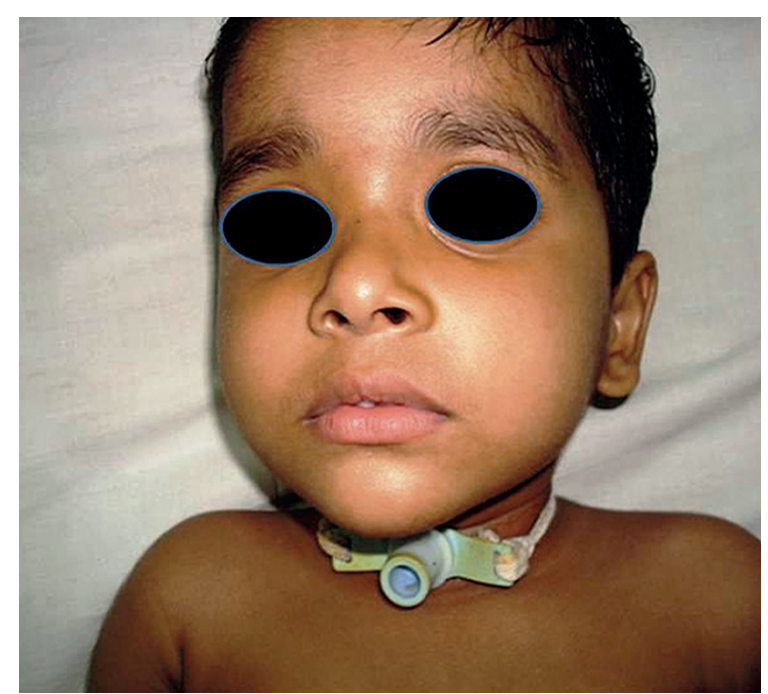

FIGURE 1. Child after tracheostomy for bilateral abductor palsy of vocal cord 


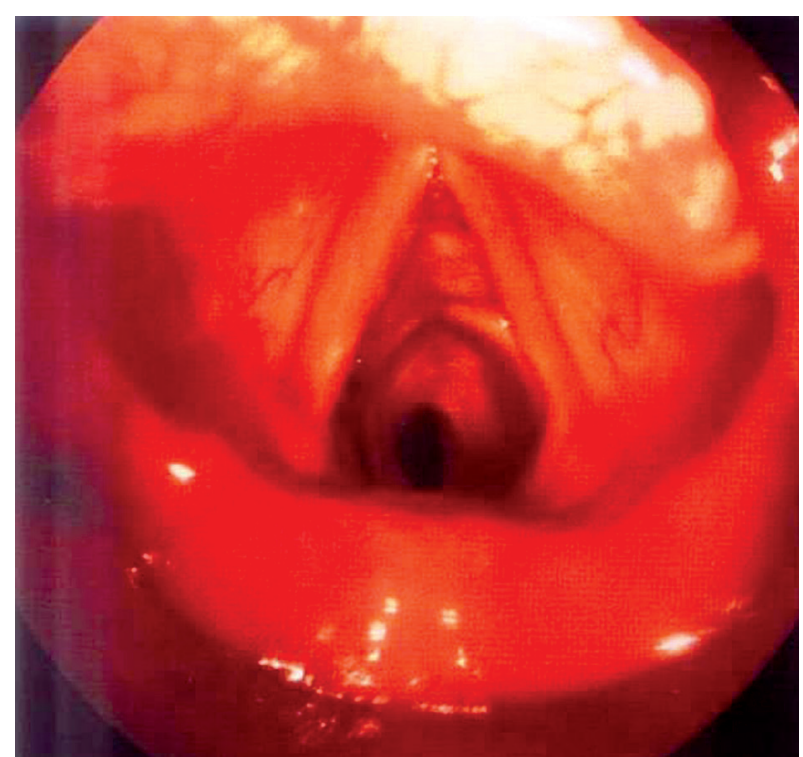

FIGURE 2. Endoscopic picture showing subglottic stenosis

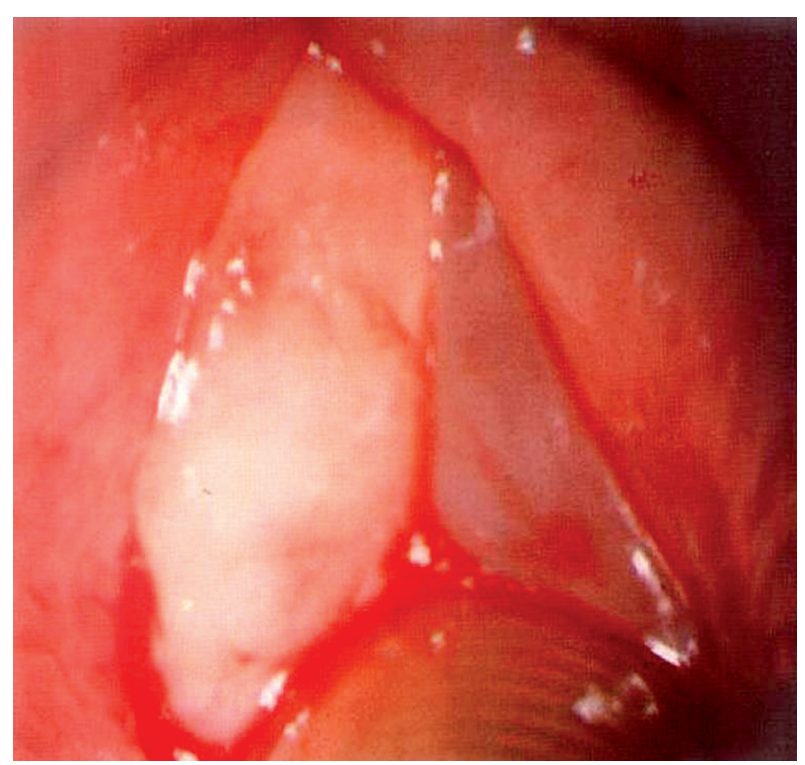

FIGURE 4. Endoscopic picture showing laryngeal papillomatosis

TABLE 1. Indications for tracheostomy

\begin{tabular}{|l|c|c|}
\hline Indications & $\begin{array}{c}\text { Number } \\
\text { of patients }(n)\end{array}$ & $\begin{array}{c}\text { Percentage } \\
(\%)\end{array}$ \\
\hline Prolonged mechanical & 68 & 72.27 \\
ventilation & 42 & 47.72 \\
Pulmonary dysfunction & 22 & 25.00 \\
Neuromuscular diseases & 4 & 4.54 \\
Infections & 20 & 22.72 \\
Airway obstruction & 7 & 7.95 \\
Subglottic stenosis & 1 & 1.13 \\
Subglottic haemangioma & 4 & 4.54 \\
Bilateral vocal cord paralysis & 3 & 3.40 \\
Head and neck neoplasms & 2 & 2.27 \\
Laryngeal papillomatosis & 2 & 2.27 \\
Laryngomalacia & 1 & 1.13 \\
Caustic ingestion & & \\
and laryngeal stenosis & \multicolumn{2}{|}{} \\
\hline
\end{tabular}

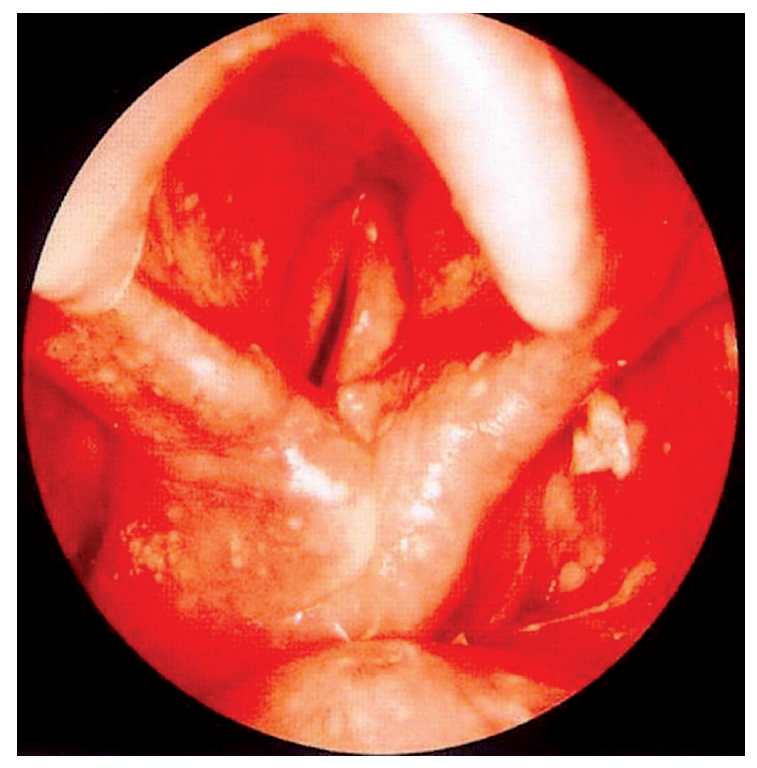

FIGURE 3. Endoscopic picture showing bilateral abductor paralysis of vocal cords

\section{RESULTS}

The total number of children in this study who underwent tracheostomy during the last 10 years was 88 . Out of the 88 children who underwent tracheostomy during the study period, 47 (53.40\%) were boys and 41 (46.59\%) were girls. The youngest child was seven months old and the oldest was 16 years old (mean age 8.8 years). None of the paediatric patients underwent tracheostomy during the neonatal period. There were nine $(10.22 \%)$ infants, 19 pre-school aged children (21.59\%), 32 school going children (36.36\%), and 28 adolescents (31.81\%) who underwent tracheostomy.

The most common indication in this study was for prolonged ventilation $(68,72.27 \%)$ followed by airway obstruction $(20,22.72 \%)$. Pulmonary pathology was the major cause for tracheostomy in the case of prolonged ventilation. There were 42 patients who underwent tracheostomy for pulmonary dysfunction, 22 children who underwent tracheostomy for neurological lesions, and four underwent tracheostomy due to infections. Subglottic stenosis was the main cause for airway obstruction. Out of all indications for tracheostomy, seven (7.95\%) had subglottic stenosis (Fig. 2) due to prolonged oro-tracheal intubation, one $(1.13 \%)$ had congenital stenosis due to subglottic haemangioma, four (4.54\%) had bilateral abductor palsy of vocal cords (Fig. 3), two (2.27\%) had laryngeal papillomatosis (Fig. 4), two (2.27\%) had laryngomalacia, three (3.40\%) had head and neck tumours, and one $(1.13 \%)$ had caustic ingestion (Table 1$)$. The children who acquired laryngotracheal stenosis had oro-tracheal intubation for 22 days (mean value).

Complications from the tracheostomy were seen in $18(20.45 \%)$ patients. Out of these 18 patients, three (16.66\%) had intra-operative bleeding, one (5.55\%) had apnoea, four $(22.22 \%)$ had cannula obstruction, two 
(11.11\%) had accidental tube displacement, two (11.11\%) had aspiration bronchopneumonia, one (5.55\%) had neck emphysema, one (5.55\%) had tracheomalacia, one (5.55\%) had stomal infection, one (5.55\%) had pneumothorax, one (5.55\%) had tracheal stenosis, and one (5.55\%) had post-decannulation trachea-cutaneous fistula (Table 2). There were no intra-operative complications.

All the tracheostomies were done successfully with appropriate tracheostomy tubes (Fig. 5) of different sizes (Table 3), except one patient who died just after one hour post-surgery.

\section{DISCUSSION}

Tracheostomy is one of the oldest surgical procedures described in medical literature. Presently, tracheostomy is a standard surgical procedure for managing long-term ventilator-dependent patients [5]. Tracheostomy in paediatric patients is often done to relive stridor and is helpful in cases of prolonged ventilation in children. The indications for paediatric tracheostomy have changed over the period of the last four decades. Initially, infectious conditions like epiglottis and laryngotracheobronchitis were common indications for tracheostomy [6]. As the treatment of infectious conditions of the airway improved, the indications for paediatric tracheostomy changed drastically. Introduction of Haemophilus influenza type B vaccine and improvements in intensive care units have reduced the number of tracheostomies for infectious diseases of the upper airways during the 1980s and 1990s [7]. Nowadays paediatric tracheostomy is commonly done for prolonged ventilation, upper airway obstruction, and neurological diseases causing hypotonia [8].

Tracheostomy protects from aspiration by giving access to the tracheobronchial toilet. Tracheostomy prevents stenosis of laryngotracheal area by avoiding long term intubation. It facilitates weaning from a ventilator by eliminating ventilator dead space. In our retrospective study, the common indications for tracheostomy among children were prolonged ventilation $(72.27 \%)$, upper airway block (22.72\%), and neurological lesions (25\%). Laryngomalacia, recurrent laryngeal papillomatosis, and laryngotracheal stenosis are common aetiologies for tracheostomy in children, which were also seen in this study. The management of laryngomalacia is based on observation and follow-up in the majority of cases because symptoms often disappear by the second year of life. In some cases of severe laryngomalacia, it has a bad outcome that needs surgical intervention. There are various surgical interventions available for the treatment of laryngomalacia, like supraglottoplasty, partial resection of the aryepiglottic folds, and epiglottopexy. In a few severe cases of laryngomalacia patients do not improve with surgical procedure and require ventilation through tracheostomy. Recurrent laryngeal papillomatosis is usually seen in children. The treatment of laryngeal papillomato-
TABLE 2. Complications of paediatric tracheostomy

\begin{tabular}{|l|c|c|}
\hline Complications & $\begin{array}{c}\text { Number } \\
\text { of patients }(\boldsymbol{n})\end{array}$ & $\begin{array}{c}\text { Percentage } \\
(\%)\end{array}$ \\
\hline Intra-operative complication & 4 & 22.22 \\
Bleeding & 3 & 16.66 \\
Apnoea & 1 & 5.55 \\
\hline Post-operative complications & 14 & 77.77 \\
Surgical neck emphysema & 1 & 5.55 \\
Tube obstruction & 4 & 22.22 \\
Accidental tube & 2 & 11.11 \\
displacement & 1 & \\
Pneumothorax & 2 & 5.55 \\
Aspiration pneumonia & 1 & 11.11 \\
Stomal infection & 1 & 5.55 \\
Tracheomalacia & 1 & 5.55 \\
Post-decannulation trachea- & & 5.55 \\
cutaneous fistula & 1 & 5.55 \\
Tracheal stenosis & \\
\hline
\end{tabular}

TABLE 3. Tracheostomy tube size. In children 2 years of age or over, the formula to calculate internal diameter of tracheostomy tube is (age in years +16$) / 4$

\begin{tabular}{|l|c|}
\hline Age of the children & Tracheostomy tube size \\
\hline Premature & 2.0 or 2.5 \\
Full term & 3.0 or 3.5 \\
6 months to 1 year & 3.5 or 4.0 \\
1 to 2 years & 4.0 \\
2 to 3 years & 4.0 or 4.5 \\
3 to 4 years & 5 \\
4 to 5 years & 5 or 5.5 \\
\hline
\end{tabular}

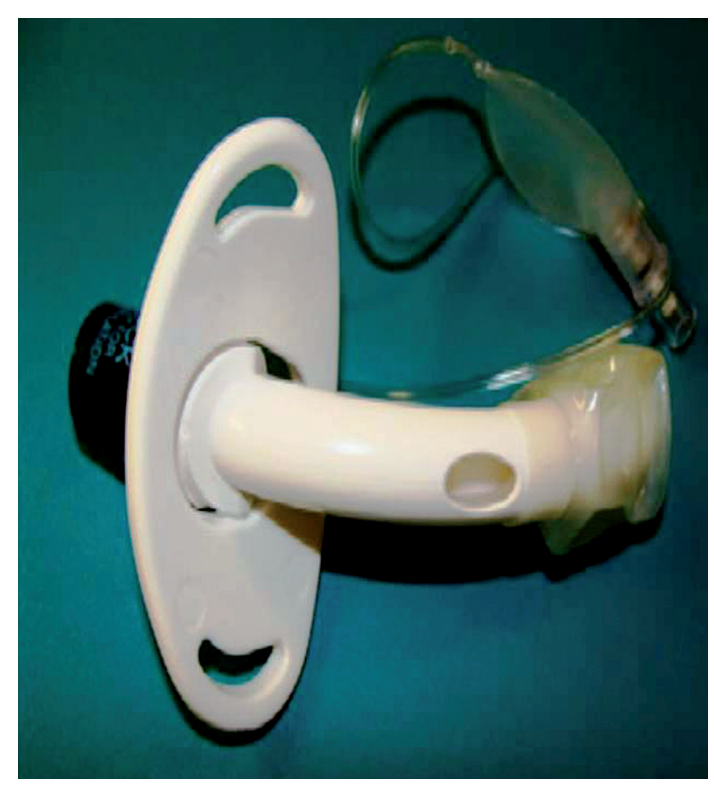

FIGURE 5. Paediatric tracheostomy tube

sis is to excise the papillomatous growth from the larynx to keep the airway patent. However, in certain cases, the lesion is highly recurrent and causes airway obstruction 
and requires tracheostomy. Upper airway obstruction can be seen in certain craniofacial malformations like Robin's sequence, Treacher-Collins syndrome, Beckwith-Wiedemann syndrome, Nager syndrome, and CHARGE association. These craniofacial anomalies with upper airway obstruction often require tracheostomy due to neurological involvement [9].

Children who need prolonged ventilator support for weeks or months require tracheostomy, which facilitates pulmonary toilet and reduces the laryngotracheal lesions like subglottic stenosis and tracheomalacia. Hence, longterm intubation leads to different morbid complications, which is one of the most important indications for tracheostomy in children [10]. The most common and dangerous complication of children with prolonged oro-tracheal intubation is subglottic stenosis. Ulcerations usually occur at the subglottic site after prolonged intubation, which leads to fibrous scar tissue formation and causes stenosis [11]. The clinical presentations of the subglottic stenosis are directly related to the degree of stenosis of the subglottic area. Grade I subglottic stenosis is usually asymptomatic, but symptoms are aggravated during upper airway infections. Grade III and IV are more severe varieties, requiring urgent tracheostomy when patients presents with air hunger, dyspnoea, and biphasic stridor. Neurological and neuromuscular diseases in children, such as spinal muscular atrophy type I, congenital myasthenic syndrome, and myotubular myopathy, may lead to compromise of the airway and the infant may unable to clear the secretions or the respiratory pump may fail, and these children may require longer ventilatory support [12].

Tracheostomy in children is more challenging than in adults because of the smaller, more pliable trachea and limited extension of the operating site. The morbidity and mortality for paediatric tracheostomy are around two to three times more than for adult patients [13]. However, a few reports described similar complications in children and adults [14]. Major complications of paediatric tracheostomies are haemorrhage, pneumothorax, and accidental decannulation. Complications are higher among paediatric patients who undergo tracheostomies [15] and vary from 5 to 49\% [16]. The complications are more frequent in children, which may be due to younger age, prolonged requirement for tracheostomy, co-morbid pathology and smaller size airway [17]. In tracheostomy patients, airflow does not occur or less air flows through the nose and nasopharynx, leading to chronic physiologic nasal congestion. There may be hypertrophy of lymphoid tissue at the nasopharynx, oropharynx, and hypopharynx. Children with tracheostomy tube are at risk of eustachian tube dysfunction due to reduced nasal airflow and absence of physiological opening of the tube at the time of swallowing. This may lead to increased risk of serous otitis media and hearing loss. Tracheostomy has some indirect side effects on the gastrointestinal system. There is increased risk of gastroesophageal reflux due to prolonged bed rest in the majority of tracheostomised children, as well as exposure to non-physiological feeding patterns. Gastroesophageal reflux can be exacerbated by constipation, mainly seen in infants and children with tracheostomies, particularly those with limited chance to perform a Valsalva manoeuvre [18].

Tracheostomy in children may negatively affect oral feeding, probably due to alterations in the mechanics of swallowing with relative tethering of the larynx, and altered sensation in the larynx and pharynx. Because there is shifting of airflow from the nose to the tracheostomy tube causing changes in the mechanics needed to initiate swallowing. There are also changes in olfaction and perception of food in tracheostomised children. One study showed $80 \%$ incidence of dysphagia among tracheostomised infants and toddlers [19]. Feeding delay among tracheostomy patients requires occupational therapy and swallowing therapy. However, many children with tracheostomy tubes need feeding tubes.

The critical period in tracheostomy is the initial period after the procedure. During this time it is often difficult to re-cannulate the tracheostomy tube after accidental displacement because the stoma or tract is not well formed and there is a risk of inserting the tube into a false tract instead of the trachea. Other immediate postoperative complications are subcutaneous emphysema or even pneumothorax, postoperative bleeding. The rates of these complications in early age infants are higher [20]. Bleeding from or around the tracheostomy site may occur due to repeated suction injury or after tracheostomy tube change where reinsertion of the tube needs some manipulation. The most common cause of bleeding around the stoma area is formation of granulation tissue at or just inside the stoma. Granulation tissue may appear as friable tissue/growth arising from the mucosal layer, which appears red to pink in colour. The granulation of the stoma area requires treatment with an antibacterial dressing, a combination of antibiotic and steroid solution, or steroid ointment applied externally at the stoma area. Some cases may need silver nitrate cautery in granulation tissue as the stoma area becomes fibrotic and behaves like keloid, which may be excised surgically.

Subglottic stenosis or acquired laryngotracheal stenosis (LTS) is a common indication for tracheostomy, particularly in children who need prolonged oro-tracheal endotracheal intubation. LTS can occur by tracheostomy tube by creating inflammation and irritation of laryngotracheal airway. Some children of LTS can be treated by balloon dilation. LTS often requires laryngotracheal reconstruction before decannulation. Prolonged tracheostomy for ventilator support may lead to tracheomalacia, which may again require prolonged tracheostomy. Tracheal stenosis in the case of prolonged tracheostomy leads to many controversies regarding its aetiology. It has been documented that underweight newborns and larger size tracheostomy tubes may lead to tracheal stenosis [21]. 
Prolonged use of a tracheostomy tube may cause the stoma to reduce into a non-functional size, although it may not close completely, which may lead to formation of trachea-cutaneous fistula. Some authors believe that the technique that is used for tracheostomy may be a cause for trachea-cutaneous fistula [22]. For example, techniques like starplasty, in which the stoma is attached to the skin using sutures to reduce pneumothorax and accidental decannulation, may need intervention to correct the trachea-cutaneous fistula [23]. Standardisation of the tracheostomy technique and proper post-tracheostomy care help to improve the quality of patient care, decrease the unnecessary laryngotracheal procedure, and reduce the economic burden of tracheostomy among paediatric patients.

Percutaneous tracheostomy is an attractive option in adult patients and is easily performed at bedside, whereas it is highly controversial in paediatric patients [24]. Complications, mortality, and cardio-respiratory arrest are higher than in classical technique in children [24]. More studies are required to establish indications and contra-indications for percutaneous tracheostomy among children.

\section{CONCLUSIONS}

Tracheostomy in paediatric patients is often indicated for prolonged ventilation. Advanced paediatric anaesthesia techniques and vaccinations among children are changing the role of tracheostomy from an emergency life-saver to a more elective surgical procedure. Proper timing and indications of paediatric tracheostomy improve the morbidity and mortality of the paediatric tracheostomy. Complications of paediatric tracheostomy are uncommon but potentially serious, and need specialised care by a multidisciplinary team with a defined patient care protocol.

\section{DISCLOSURE}

The authors declare no conflict of interest.

\section{REFERENCES}

1. Ligoski Dal'Astra AP, Quirino AV, de Sousa Caixêta JA, Avelino MAG. Tracheostomy in childhood: review of the literature on complications and mortality over the last three decades. Braz J Otorhinolaryngol 2017; 83: 207-214.

2. Özmen S, Özmen Ö. Pediatric tracheostomies: a 37-yearexperience in 282 children. Int J Pediatr Otorhinolaryngol 2009; 73: 959-961.

3. Yellon R, Maguire R, Tuchman J. Paediatric tracheotomy. In: Tracheotomy Management: A Multidisciplinary Approach. Cambridge University Press, New Delhi 2012: 72-86.

4. Itamoto CH, Lima BT, Sato J, Fuijita RR. Indications and complications of tracheostomy in children. Braz J Ootorhinolaryngol 2010; 76: 326-331.

5. Swain SK, Behera IC, Sahu MC. Bedside open tracheostomy at intensive care unit-Our experiences of 1000 cases at a tertiary care teaching hospital of eastern India. Egyptian J of Ear Nose Throat Allied Sci 2017; 18: 49-53.
6. Wetmore RF, Handler SD, Potsic WP. Pediatric tracheostomy: experience during the past decade. Ann Otol Rhinol Laryngol 1982; 91: 628-632.

7. Ozmen S, Ozmen OA, Unal OF. Pediatric tracheotomies: a 37-year experience in 282 children. Int J Pediatr Otorhinolaryngol 2009; 73: 959-961.

8. Carron JD, Derkay CS, Strope GL, et al. Pediatric tracheotomies: changing indications and outcomes. Laryngoscope 2000; 110: 10991104.

9. Itamoto CH, Lima BT, Sato J, Fujita RR. Indications and Complications of Tracheostomy in Children. Braz J Otorhinolaryngol 2010; 76: 326-331.

10. Parrilla C, Scarano E, Guidi ML, et al. Current trends in paeditric tracheostomies. Int J Pediatr Otorhinolaryngol 2007; 71: 1563-1567.

11. Swain SK, Sahu MC, Choudhury J. Subglottic stenosis in pediatric patients. Pediatr Pol 2018; 93: 80-85.

12. Benson RC, Henson KA, Gildengoren G, Hsia D. International survey of physician recommendation for tracheostomy for spinal muscular atrophy type I. Pediatr Pulmonol 2012; 47: 606-611.

13. Kremer B, Botos-Kremer AI, Eckel HE, Schlondorff G. Indications, complications, and surgical techniques for pediatric tracheostomies-an update. J Pediatr Surg 2002; 37: 1556-1562.

14. Suslu N, Ermutlu G, Akyol U. Pediatric tracheotomy: comparison of indications and complications between children and adults. Turk J Pediatr 2012; 54: 497-501.

15. Carr MM, Poje CP, Kingston L, et al. Complications in pediatric tracheostomies, Laryngoscope 2001; 111: 1925-1928.

16. Serra A, Cocuzza S, Longo MR, et al. Tracheostomy in childhood: new causes for an old strategy. Eur Rev Med Pharmacol Sci 2012; 16: $1719-1722$.

17. Gianoli GJ, Miller RH, Guarisco JL. Tracheotomy in the first year of life. Ann Otol Rhinol Laryngol 1990; 99: 896-901.

18. DeMauro SB, Wei JL, Lin RJ. Perspectives on neonatal and infant tracheostomy. Semin Fetal Neonatal Med 2006; 21: 285-291.

19. Norman V, Louw B, Kritzinger A. Incidence and description of dysphagia in infants and toddlers with tracheostomies; a retrospective review. Int J Pediatr Otorhinolaryngol 2007; 71: 1087-1092.

20. Corbett HJ, Mann KS, Mitra I, et al. Tracheostomy - a 10-year experience from a UK pediatric surgical center. J Pediatr Surg 2007; 42: 1251-1254.

21. Viswanathan S, Mathew A, Worth A, Mhanna MJ. Risk factors associated with the need for a tracheostomy in extremely lowbirth weight infants. Pediatr Pulmonol 2013; 48: 146-150.

22. Parrilla A, Scarano E, Guidi ML, et al. Currenttrends in paediatric tracheostomies. Int J Pediatr Otorhinolaryngol 2007; 71: 1563-1567.

23. Solares CA, Krakovitz P, Hirose K, Koltai PJ. Starplasty: revisiting a pediatric tracheostomy technique. Otolaryngol Head Neck Surg 2004; 131: 717-722.

24. Dulguerov P, Gysin C, Pernerger T, Chevrolet J-C. Percutaneous or surgical tracheostomy: a meta-analysis. Crit Care Med 1999; 27: $1617-1625$ 